\title{
Peony seed oil protects against obesity-induced non- alcoholic fatty liver disease via modulation of endoplasmic reticulum stress
}

\author{
Jianhui Su, Jiejie Tao, Longhua Xing, Chengshuang Ao, Yuxuan Ma, Juncai \\ Wan, Zhe Tang* \\ School of Marine and Bioengineering, Yancheng Institute of Technology, Yancheng 224051, PR China
}

*For correspondence: Email: tangzhe1983@163.com

Sent for review: 27 December 2020

Revised accepted: 27 February 2021

\begin{abstract}
Purpose: To investigate the effect of Peony seed oil (PO) on non-alcoholic fatty liver disease (NAFLD), and the underlying mechanism of action.

Methods: A mouse model of NAFLD was established using high-fat diet (HFD) for 12 weeks. Serum levels of triacylglycerol (TG), total cholesterol (TC), glucose and free fatty acids (FFAs) were determined. Activities of aspartate aminotransferase (AST) and alanine amino transferase (ALT) were assayed in liver homogenates, while mRNA and protein expressions of GRP78, x-box binding protein 1 (XBP1) and phosphorylated inositol-requiring enzyme 1 ( $p$-IRE1 $\alpha$ ) were assayed in liver tissue using real-time quantitative polymerase chain reaction (qRT-PCR) and Western blotting, respectively. Expression levels of tumor necrosis factor- $\alpha$ (TNF- $\alpha$ ), interleukin-6 (IL-6) and interleukin-1 $\beta$ (IL-1 $\beta)$ were also determined by $q R T-P C R$.

Results: HFD diet led to significant increases in body weight as well as serum TG, TC, glucose and FFA levels. However, treatment with PO significantly reversed the effect of HFD on these parameters $(p<0.05)$. The mRNA expression levels of TNF- $\alpha$, IL-6 and IL-1 $\beta$ were significantly higher in HFD control group than in normal control group, but were significantly reduced after $P O$ treatment $(p<0.05)$. The protein expression levels of GRP78, XBP-1 and $p$-IRE1a significantly increased in the liver of NAFLD group, when compared with normal control rats, but were significantly downregulated by treatment with $P O(p<0.05)$.

Conclusion: PO attenuates HFD-induced NAFLD via inactivation of endoplasmic reticulum stress (ERS) pathway. Thus, PO has potentials for the treatment of NAFLD but further studies are required to ascertain this.
\end{abstract}

Keywords: Non-alcoholic fatty liver disease (NAFLD), Peony seed oil, endoplasmic reticulum stress (ERS), Inflammatory cytokines, High-fat diet

This is an Open Access article that uses a fund-ing model which does not charge readers or their institutions for access and distributed under the terms of the Creative Commons Attribution License (http://creativecommons.org/licenses/by/4.0) and the Budapest Open Access Initiative (http://www.budapestopenaccessinitiative.org/read), which permit unrestricted use, distribution, and reproduction in any medium, provided the original work is properly credited.

Tropical Journal of Pharmaceutical Research is indexed by Science Citation Index (SciSearch), Scopus, International Pharmaceutical Abstract, Chemical Abstracts, Embase, Index Copernicus, EBSCO, African Index Medicus, JournalSeek, Journal Citation Reports/Science Edition, Directory of Open Access Journals (DOAJ), African Journal Online, Bioline International, Open-J-Gate and Pharmacy Abstracts

\section{INTRODUCTION}

Non-alcoholic fatty liver disease (NAFLD), a metabolic syndrome characterized by liver TG levels exceeding $5 \%$ by weight in the absence of excessive alcohol consumption (<20 g per day), is closely related to insulin resistance, obesity and diabetes mellitus [1]. The spectrum 
of NAFLD diseases ranges from steatosis to non-alcoholic steatohepatitis (NASH). In severe cases, liver failure or cancer may develop [2]. Several factors involved in the development of NAFLD lead to the accumulation of liver fat. Impairment of lipid metabolism induces $\mathrm{NASH}$, oxidative stress, inflammation, and hepatic injury [3].

However, the mechanisms underlying the etiology of NAFLD are yet to be fully understood. A "two-hit" hypothesis has been proposed to explain the pathogenesis of $\mathrm{NASH}$. In the first 'hit', fats accumulate in the liver. Then, the resultant steatosis sensitizes the hepatocytes to additional proinflammatory insults (second 'hit'), aggravating liver fibrosis, and ultimately leading to NAFLD [4]. Endoplasmic reticulum (ER) stress is attributed to accumulation of unfolded or misfolded proteins in the cell, resulting in induction of type 2 diabetes, obesity and liver cancer. Cells form unfolded protein response (UPR) against ER stress for restoration of homeostasis. The formation of UPR is carried out by three ER transmembrane receptors: inositol-requiring enzyme 1 (IRE1 $1 \alpha)$, PKR-like ER kinase (PERK) and activating transcription factor (ATF6) [5].

Activation of IRE1 1 induces the splicing of end pieces of XBP-1 mRNA required for normal fatty acid synthesis in the liver. It has been reported that the XBP1 protein expression in mice corresponds to the induction of critical genes involved in fatty acid synthesis, such as sterolregulatory element binding protein (SREBP-1), fatty acid synthase (FAS) and acetyl-CoA carboxylase (ACC) [6]. In addition, studies have shown that ER stress contributes to inflammatory response by activating NF-kB. The activated NF-KB upregulates the production of interleukin (IL)-1 $\beta$, IL-6, and TNF $\alpha$ associated with NASH [7]. The upregulation of transcripts of ER stress is correlated with inflammasome priming and liver fat accumulation in NASH patients. This highlights their relevance in development of the disease. Existing evidence reveals that $\mathrm{N}-3$ polyunsaturated fatty acids (PUFAs) modulate ER stress and attenuate NAFLD via the regulation of lipid metabolism or alleviation of hepatic inflammation [8].

Peony seed oil (PO) is a new food resource for high level $\alpha$-linolenic acid (>38\%) in China. It has been observed that PO supplementation significantly lowers hepatic cholesterol and triglycerides, and diminishes accumulation of hepatic droplets.
Previous studies have reported that $\mathrm{PO}$ significantly lowered hepatic cholesterol and triglyceride levels [9]. However, the protective effect of $\mathrm{PO}$ on NAFLD, and the underlying mechanisms have not been investigated. The present study was carried out to investigate the possible protective effect of PO against NAFLD, and to identify the underlying molecular mechanisms.

\section{EXPERIMENTAL}

\section{Materials}

Commercial kits used for determination of AST, ALT, TG and TC, as well as total RNA isolation reagents were purchased from Nanjing Jiancheng Bioengineering Institute (Nanjing, China). Peony seed oil was obtained from Anhui Tongling Ruipu Peony Industry Development Co. Ltd in China. All solvents and reagents were obtained from Sinopharm Chemical Reagent Co. Ltd. (Shanghai, China), and were of analytical grade. This study was carried out in accordance with the National Institutes of Health Laboratory Animal Care and Use Guidelines [10]. The study protocol was approved by the Jiangsu Vocational College of Medicine Animal Use and Care Committee (no. SYXK2018-0008).

\section{Animals and treatment regimen}

Six-week-old male C57BL/6J mice were purchased from Shanghai Slac Laboratory Animal Co. Ltd. (Shanghai, China). All mice were maintained in a room with a 12-h light/12-h dark cycle under controlled temperature of $23 \pm$ $2{ }^{\circ} \mathrm{C}$ and relative humidity of $50-70 \%$. After 1 week of adaptation to the laboratory conditions, the mice were fed with either standard rodent chow (control group) with $10 \mathrm{kcal} \%$ fat, or a high-fat diet (HFD) with $45 \mathrm{kcal} \%$ fat and $2 \%$ cholesterol (w/w) for 8 weeks. In the subsequent 8 weeks, mice in the control group were kept on rodent chow, while mice on HFD were redistributed into three groups maintained on HFD, HFD+PO (10\% PO (w/w) + $45 \mathrm{kcal} \%$ fat + $2 \%$ cholesterol, or HFD+ $10 \%$ fish oil (FO, w/w) $+45 \mathrm{kcal} \%$ fat $+2 \%$ cholesterol). At the end of the experiment, the mice were fasted for $12 \mathrm{~h}$ and sacrificed via $\mathrm{CO}_{2}$ asphyxiation [11].

\section{Assessment of body and liver weights}

At the beginning and end of the experiment, the body weights of mice were recorded. The livers were excised at the end of the experiment and weighed. 


\section{Measurement of serum parameters}

Serum TC, TG, FFAS and blood glucose were determined using commercial kits according to the manufacturers' instructions. Blood was collected through cardiac puncture and centrifuged at $1000 \mathrm{G}$ for 10 min at $4{ }^{\circ} \mathrm{C}$ to obtain serum samples.

\section{Hepatic biochemical assays}

Hepatic tissues (300 mg) were homogenized in ice cold saline $(9 \mathrm{~mL})$ and centrifuged at 2500 rpm for $10 \mathrm{~min}$. The activities of AST and ALT, as well as levels of TG were determined in the supernatants. ELISA kits were used for determination of the concentrations of TNF- $\alpha$, IL-1 $\beta$, and IL-6.

\section{Total mRNA extraction and real-time quantitative PCR}

Total hepatic RNA was isolated from liver using TRIzol reagent (Biomiga). The RNA was reverse-transcribed to cDNA using M-MLV reverse transcriptase. The qPCR assays were performed in a $10-\mu \mathrm{L}$ reaction mixture containing cDNA, gene-specific primers and SYBR Green I PCR Master Mix (Takara, Dalian, China). Reactions were performed in duplicate in an $A B I$ PRISM 7000 Sequence Detection System (Applied Biosystems). The amplification reactions were carried out under the following PCR conditions: cDNA was initially denatured for $5 \mathrm{~min}$ at $94{ }^{\circ} \mathrm{C}$, followed by 40 repeats at 95 ${ }^{\circ} \mathrm{C}$ for $15 \mathrm{sec}, 60^{\circ} \mathrm{C}$ for $30 \mathrm{sec}$, and $72{ }^{\circ} \mathrm{C}$ for 30 sec. The purity of PCR products was verified using melting curve and agarose gel electrophoresis, and the comparative threshold cycle (Ct) method $\left(2^{-\Delta \Delta C t}\right)$ was used to calculate the relative expression levels of the target genes, with GAPDH as the standard gene [12].

\section{Western blot analysis}

Proteins were extracted from liver tissues and quantified using the BCA method. Equal amounts of proteins were separated on SDSpage gels and transferred to polyvinylidene difluoride (PVDF) membranes. The membranes were blocked with $5 \%$ non-fat milk and incubated overnight with primary antibodies against XBP1, GRP78 and PIRE-1A at $4{ }^{\circ} \mathrm{C}$, followed by incubation with horseradish peroxidase (HRP)-conjugated secondary antibody. B-actin was used as internal control. The protein bands were visualized and detected using chemiluminescence image system (BIORAD).

\section{Statistical analysis}

All data are expressed as mean \pm SD. Statistical analysis of data was carried out with one-way ANOVA and Fisher's PLSD test using SPSS 16.0 for Windows (SPSS Inc., Chicago, IL, USA). Values of $p<0.05$ were regarded as statistically significant.

\section{RESULTS}

\section{Body weight and feed consumption}

The general physiological characteristics of experimental groups are presented in Figure 1. As shown in Figure $1 \mathrm{~A}$, there were no significant differences among the HF, PO and FO groups with regard to feed consumption $(p<$ $0.05)$. However, higher feed intake was seen in the control group. As expected, body weight of mice fed with HFD for 16 weeks was significantly higher than that of mice in the control, PO or FO group $(p<0.05)$. The mean body weight of mice fed HFD was increased by $20.37 \mathrm{~g}$, whereas that of mice in the control group increased by $4.35 \mathrm{~g}$ (Figure $1 \mathrm{~B}$ ). The final mean body weights of the groups treated with FO and PO increased by 12.58 and $13.31 \mathrm{~g}$, respectively. The mean body weight of mice treated with $\mathrm{FO}$ was lower than that of mice in the PO group, although the difference was not significant.

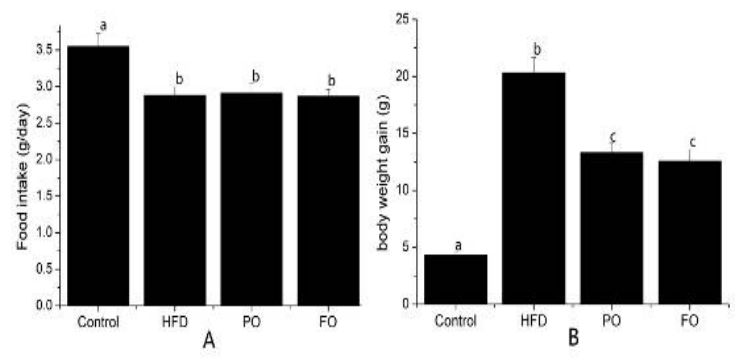

Figure 1: Effects of $\mathrm{PO}$ and $\mathrm{FO}$ on feed consumption and body weight of C57BL/6J mice fed HFD, normal diet or HFD along with PO or FO. Data are presented as mean $\pm S D(n=10)$. Means with different letters differ significantly $(p<0.05$; Fisher's PLSD test. (A) Feed intake, and (B) body weight in mice

\section{Serum biochemical parameters}

As shown in Figure 2, HFD significantly increased the serum levels of glucose, FFAs, TG, and TC, when compared to the control group. The PO-treated groups had significant decreases in levels of TG, TC, glucose and FFAs, relative to mice fed with HFD diet. There were no statistically significant differences 
between the control group and FO group, with respect to lipid parameters.

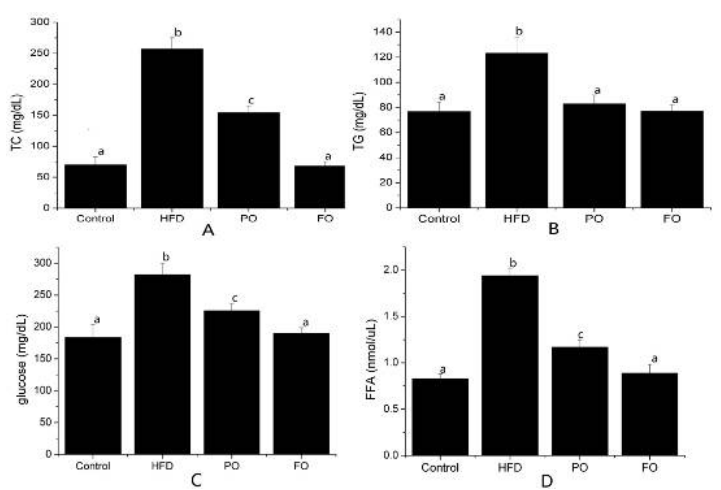

Figure 2: Effects of $\mathrm{PO}$ and $\mathrm{FO}$ on serum levels of glucose, FFAs, TC and TC in C57BL/6J mice fed HFD, normal diet or HFD, with PO or FO. Data are presented as mean \pm SD $(n=10)$. Means with different letters differ significantly $(p<0.05$; Fisher's PLSD test)

\section{Hepatic biochemical parameters}

Figure 3 A shows that the liver weight of HFDfed mice was 2.2 folds higher than that of mice in the control group. Supplementation with $\mathrm{PO}$ or FO significantly suppressed the increase in liver weight, when compared to HFD group. Furthermore, the levels of TG in HFD group was significantly increased. However, the increase was reversed by treatment with $\mathrm{PO}$ and $\mathrm{FO}$. Compared to HFD group, $\mathrm{PO}$ and FO significantly decreased levels of AST by 49.7 and $54.25 \%$, respectively. Similarly, supplementation with $\mathrm{PO}$ and $\mathrm{FO}$ markedly suppressed the levels of ALT in the liver tissue by 85.8 and $87.0 \%$, respectively $(p<0.05)$.

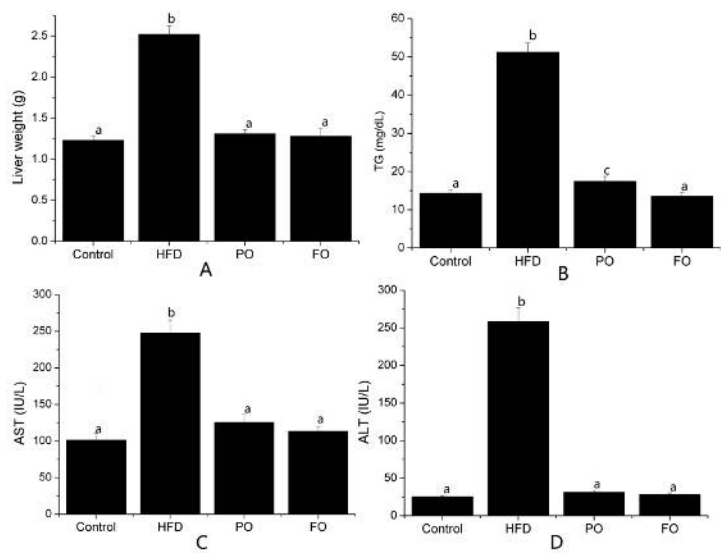

Figure 3: Effect of PO or FO supplementation on liver biochemical parameter levels in mice fed HFD, normal diet or HFD, with PO or FO. Data are presented as mean \pm SD $(n=10)$. Means with different letters differ significantly $(P<0.05$; Fisher's PLSD test)

\section{Levels of hepatic inflammatory cytokines}

Figure 4 shows that the levels of inflammatory cytokines TNF- $\alpha, \mathrm{IL}-1 \beta$ and IL- 6 were increased in group HFD ( $p<0.05$, group HFD versus group control) and significantly reduced in groups $\mathrm{PO}$ and FO ( $p<0.05$, groups $\mathrm{PO}$ and FO versus group HFD) which were upregulated in NAFLD rats.

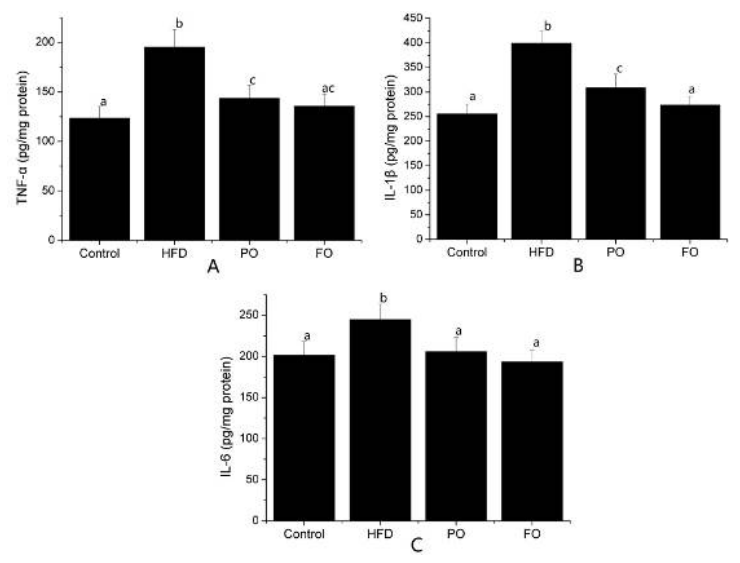

Figure 4: Effect of $\mathrm{PO}$ or $\mathrm{FO}$ supplementation on hepatic inflammatory cytokine levels in mice fed HFD, normal diet or HFD, with PO or FO. Data are presented as mean $\pm S D(n=10)$. Means with different letters differ significantly $(p<0.05$; Fisher's PLSD test)

\section{Hepatic endoplasmic reticulum stress}

The expression levels of proteins involved in ER stress in the liver are shown in Figure 5 . The expression levels of GRP78, p-IRE1a and its targets XBP1 in HFD-fed mice were 1.8, 3.3, and 2.5-fold, respectively, higher than the corresponding expression levels in the control group. When compared to those of mice in the HFD-fed group, the levels of GRP78, pIRE-1a and XBP1 were significantly down-regulated by $\mathrm{PO}$ and $\mathrm{FO}$ treatments.

\section{DISCUSSION}

Non-alcoholic liver disease (NAFLD) is a complication of metabolic syndrome (obesity, hyperglycemia, dyslipidemia and hypertension) [13]. Based on previous studies, NAFLD was successfully induced in C57BL/6J mice using HFD with $45 \mathrm{kcal} \%$ fat. The mean weight of mice fed with HFD was significantly higher than that of mice in control group. This indicates that HFD, obesity and NAFLD are closely related. It was found that serum glucose, TC, TG and FFAs, as well as hepatic TG, AST, and ALT 
levels, and liver weight were significantly increased. Disorders of lipid metabolism contribute to the pathogenesis of NAFLD.

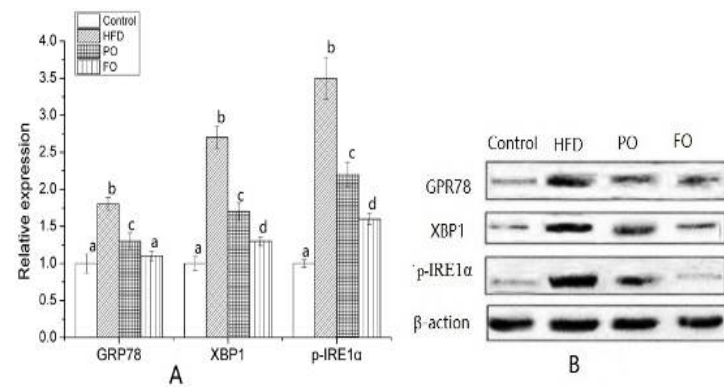

Figure 5: Effect of $\mathrm{PO}$ or $\mathrm{FO}$ supplementation on hepatic endoplasmic reticulum stress levels in mice fed HFD, normal diet or HFD, with PO or FO by Q-PCR (Figure 5a) and western-blot (Figure $5 \mathrm{~b}$ ). Data are presented as mean \pm SD ( $\mathrm{n}=10)$. Means with different letters differ significantly $(P<0.05$; Fisher's PLSD test)

Studies have shown that a reduction in FFA absorption in subjects result in elevated FFA entry into the liver, leading to increased expressions of lipogenesis markers involved in fatty acid and TG synthesis [14]. The excess fatty acids are deposited in the liver in the form of TG, resulting in NAFLD. In the present study, administration of $\mathrm{PO}$ decreased the levels of FFAs. The results indicated that PO prevented hepatic lipid accumulation by reducing serum FFA levels and regulating lipid metabolism in long-term HFD-fed mice. In addition, moderate levels of ER stress and inflammation were induced.

Earlier studies revealed the development of NAFLD in combination with oxidative stress and inflammatory cytokines [15]. However, the protective effect of polyunsaturated fatty acids against ER stress and associated hepatic steatosis has not been reported. In this study, it was demonstrated for the first time that PO, which is rich in omega- 3 polyunsaturated fatty acids, attenuated ER stress and prevented development of NAFLD. Long-term activation or unresolved ER stress has emerged as a major contributor to cases of NAFLD [16]. Therefore, pharmacological modulators of ER stress could be therapeutic candidates for NAFLD.

Excessive intake of dietary fats makes the liver vulnerable to ER stress, leading to hepatocyte inflammation, pro-inflammatory cytokines, and hepatocyte mitochondrial dysfunction. It has been revealed that tazoramide has a high therapeutic potential for NAFLD and related diseases via a mechanism involving reduction of ER stress [17]. In the present study, PO and FO supplementation decreased the levels of proinflammatory cytokines (TNF- $\alpha, \mathrm{IL}-1 \beta$, and IL-6) in the liver, resulting in diminished ER stress through decreases in levels of GRP78, pIRE-1a and XBP1 in HFD-fed mice. It is well known that GRP78 is a marker of ER stress. Western blotting results showed that HFD treatment increased GRP78 level, while PO treatment reduced it. A previous study showed that flaxseed oil markedly decreased levels of proinflammatory markers (IL-1 $\beta$, TNF- $\alpha$, plkBa, plKK $\beta$ ) and unfolded protein response markers (ATF6 and GRP78) in the aorta. In the study, the expression of GRP78 was decreased in the PO group, when compared to the HFD group. This is similar to the finding in a previous study [18].

\section{CONCLUSION}

The results of this study provide, for the first time, a series of evidence showing that $P O$ reduces ER stress and inflammation in NAFLD-induced mice. Thus, PO has hepatoprotective potentials for the therapeutic for the therapeutic management of inflammations in humans.

\section{DECLARATIONS}

\section{Acknowledgement}

This work was supported by Talent Instruction Project of Yancheng Institute of Technology (nos. XJ201724 and KJC2014015).

\section{Conflict of interest}

No conflict of interest is associated with this work.

\section{Contribution of authors}

The authors declare that this work was done by the authors named in this manuscript, and all liabilities pertaining to claims relating to the content of this article will be borne by them. All authors read and approved the manuscript for publication.

\section{Open Access}

This is an Open Access article that uses a funding model which does not charge readers or their institutions for access and distributed under the terms of the Creative Commons Attribution License (http://creativecommons.org/licenses/by/ 4.0) and the Budapest Open Access Initiative (http://www.budapestopenaccessinitiative.org/rea d), which permit unrestricted use, distribution, 
and reproduction in any medium, provided the original work is properly credited.

\section{REFERENCES}

1. Machado $M$, Cortez $H$. Non-alcoholic steatohepatitis and metabolic syndrome. CURR OPIN CLIN NUTR 2006; 9(5): 637-42.

2. Mohamed HA, Emmanuel OA, Christopher DB. NonAlcoholic Fatty Liver Disease (NAFLD): new challenge for general practitioners and important burden for health authorities? PRIM CARE DIABETES 2010; 4(3): 129-37.

3. Robert SR, Carmen L. Nonalcoholic Fatty Liver Disease and the Metabolic Syndrome. NUTR CLIN PRACT 2013; 28(1): 40-51.

4. Day CP, James OF. Steatohepatitis: A tale of two Hits'? GASTROENTEROLOGY 1998; 114(4): 842-5.

5. $X u$ C, Bailly-Maitre B, Reed JC. Endoplasmic reticulum stress: cell life and death decisions. J Clin Invest 2005; 115(10)10: 2656-2664.

6. Lee AH, Scapa EF, Cohen DE, Glimcher LH. Regulation of hepatic lipogenesis by the transcription factor XBP1. Science 2008; 320(5882): 1492-1496.

7. Ribeiro PS, Cortez-Pinto H, Sola S, Castro RE, Ramalho RM, Baptista A, Moura MC, Camilo ME, Rodrigues CM. Hepatocyte apoptosis, expression of death receptors, and activation of NF-kappaB in the liver of nonalcoholic and alcoholic steatohepatitis patients. Am J Gastroenterol 2004; 99(9): 1708-1717.

8. Scorletti E, Byrne CD. Omega-3 Fatty Acids, Hepatic Lipid Metabolism, and Nonalcoholic Fatty Liver Disease. Annu Rev Nutr 2013; 33: 231-248.

9. SU J, MA C, LIU C, GAO C, NIE R, WANG $H$. Hypolipidemic Activity of Peony Seed Oil Rich in alinolenic, is Mediated Through Inhibition of Lipogenesis and Upregulation of Fatty Acid $\beta$-Oxidation. J FOOD SCI 2016; 81(4): H1001-09.

10. Council NR: Guide for the care and use of laboratory animals: National Academies Press; 2010.

11. Lai YS, Chen WC, HO CT, Lu KH, Lin SH, Tseng HC, Lin SY, Sheen LY. Garlic essential oil protects against obesity-triggered nonalcoholic fatty liver disease through modulation of lipid metabolism and oxidative stress. $J$ AGR FOOD CHEM 2014; 62(25): 5897-906.

12. Lai S, Li Y, Kuang Y, Cui H, Yang Y, Sun W, Liu K, Chen $D$, Yan $Q$, Wen L. PKC $\delta$ silencing alleviates saturated fatty acid induced ER stress by enhancing SERCA activity. Bioence Reports 2017 ; 37(6): BSR20170869.

13. Machado M, Cortez-Pinto H. Non-alcoholic steatohepatitis and metabolic syndrome. Curr Opin Clin Nutr 2006; 9(5): 637-642.

14. Serviddio G, Bellanti F, Vendemiale G. Free radical biology for medicine: learning from nonalcoholic fatty liver disease. Free Radical Biol. Med 2013; 65: 952-968.

15. Ze C, Ruifeng T, Zhigang S, Jingjing C. Role of oxidative stress in the pathogenesis of nonalcoholic fatty liver disease. Free Radical Bio Med 2020; 152: 116-41.

16. Li J, Huang J, Li JS, Chen H, Huang $K$ \& Zheng $L$. Accumulation of endoplasmic reticulum stress and lipogenesis in the liver through generational effects of high fat diets. J Hepatol 2012; 56(4): 900-907.

17. Bagci R, Sahinturk V, Sahin E. Azoramide ameliorates fructose-induced nonalcoholic fatty liver disease in mice. Tissue Cell 2019; 59: 62-69.

18. Moura-Assis A, Afonso M, Oliveira V, Morari J, Santos G, Koike $M$, Lottenberg A, Catharino R, Velloso L, Silva $A D$. Flaxseed Oil Rich in Omega-3 Protects Aorta Against Inflammation and Endoplasmic Reticulum Stress Partially Mediated by GPR120 Receptor in Obese, Diabetic and Dyslipidemic Mice Models. J NUTR BIOCHEM 2017; 53: 9-19. 